Int. J. Contemp. Math. Sciences, Vol. 2, 2007, no. 6, $275-278$

\title{
Extention of Topological Inner Invariant Means
}

\author{
Reza Memarbashi \\ Department of Mathematics, Faculty of Sciences \\ Semnan University, P.O. Box 35195-363, Semnan, Iran
}

\begin{abstract}
For a locally compact group $G$, we prove that a topological inner invariant mean on $L U C(G)$ has an extension to a topological inner invariant mean on $L^{\infty}(G)$.
\end{abstract}

Mathematics Subject Classification: 43A07, 43A10

Keywords: Inner amenability, Topological inner invariant means

\section{Introduction}

Let $G$ be a locally compact group with identity $e$ and left Haar measure $d x$. Let $L^{\infty}(G)$ and $L^{1}(G)$ denote the spaces of essentially bounded functions and integrable functions respectively.

For a function $f: G \rightarrow \mathbb{C}$ we put ${ }_{x} f(y)=f(x y)$ and $f_{x}(y)=f(y x)$ for all $x, y \in G$. Recall that a mean $m$ on a linear subspace $X$ of $L^{\infty}(G)$ containing the constants is a linear functional such that $\|m\|=m(1)=1$. If ${ }_{x} f, f_{x} \in X$ for all $f \in X$ and $x \in G$, we say that $m$ is inner invariant if $m\left({ }_{x} f\right)=m\left(f_{x}\right)$ for all $x \in G$ and $f \in X$.

We denote by $P(G)$ the set of all $\varphi \in L^{1}(G)$ with $\varphi \geq 0$ and $\|\varphi\|_{1}=1$. If $X$ is a linear subspace of $L^{\infty}(G)$ containing the constants, $X$ is said to be topological left (resp. right) invariant if $P(G) * X \subset X$ (resp. $X * P(G)^{\sim} \subset X$ ). Here $\tilde{\varphi}$ is the function defined by $\tilde{\varphi}(x)=\varphi\left(x^{-1}\right)$ for all $x \in G$ and $*$ denotes the convolution product of functions on $G$. Let $X$ be topological invariant (i.e. topological left and right invariant). A mean defined on $X$ is said to be topological inner invariant if

$$
m\left(\frac{1}{\Delta} \tilde{\varphi} * f\right)=m(f * \tilde{\varphi}) \text { for all } f \in X \text { and } \varphi \in P(G)
$$


where $\Delta$ is the modular function of $G$. The concept of topological inner invariant means (TIIM) was introduced and studied by Nasr-Isfahani [8] for a large class of Banach algebras containing $L^{1}(G)$ known as Lau algebras.

In [7] authors study locally compact groups for which $L^{\infty}(G)$ has a TIIM whose restriction to $C_{b}(G)$ is not $\delta_{e}$. In this paper we show that a TIIM on $L U C(G)$ (space of left uniformly continuous functions on $G$ ) has a topological inner invariant extension to $L^{\infty}(G)$. Also based on the technic used in [7, Th.3.2] we give a simpler proof of the well known fact that amenability implies topological amenability (see [9]).

\section{Topological inner invariant means}

It is well known [7] that any extension $E$ of $\delta_{e}$ from $C_{b}(G)$ to a mean on $L^{\infty}(G)$ is a mixed identity of $L^{\infty}(G)^{*}$; that is

$$
E\left(\frac{1}{\Delta} \tilde{\varphi} * f\right)=E(f * \tilde{\varphi})=\varphi(f) \text { for all } f \in L^{\infty}(G) \text { and } \varphi \in P(G) .
$$

In particular, $E$ is a TIIM on $L^{\infty}(G)$; here we give another proof of this fact.

Lemma 1. Let $\left(e_{i}\right)$ be a bounded approximate identity of $L^{1}(G)$ and $e_{i} \rightarrow E$ $\left(w k^{*}\right)$, then $E \in L^{\infty}(G)^{*}$ is a TIIM and $E(f)=f(e)$ for $f \in C B(G)$.

Proof. Let $\varphi \in P(G), f \in L^{\infty}(G)$, with the aid of the relations $f \cdot \varphi=$ $\left(\frac{1}{\Delta} \tilde{\varphi}\right) * f$ and $\varphi \cdot f=f * \tilde{\varphi}$ we have

$$
E\left(\left(\frac{1}{\Delta} \tilde{\varphi}\right) * f\right)=E(f . \varphi)=\lim _{i} e_{i}(f \cdot \varphi)=\lim _{i} f \cdot \varphi\left(e_{i}\right)=\lim _{i} f\left(\varphi * e_{i}\right)=f(\varphi)
$$

and

$$
E(f * \tilde{\varphi})=E(\varphi \cdot f)=\lim _{i} e_{i}(\varphi \cdot f)=\lim _{i} \varphi \cdot f\left(e_{i}\right)=\lim _{i} f\left(e_{i} * \varphi\right)=f(\varphi)
$$

And hence $E\left(\left(\frac{1}{\Delta} \tilde{\varphi} * f\right)=E(f * \tilde{\varphi})\right.$. By [6,Th.2] $E(f)=f(e)$ for $f \in C B(G)$.

For each locally compact group $G, L^{1}(G)$ has a bounded approximate identity, therefore by the above lemma and theorem of Banach Alaoghlu, $L^{\infty}(G)$ always has a topological inner invariant mean.

Theorem 2. Let $G$ be a locally compact group. Then each TIIM on $L U C(G)$ has a topological inner invariant extention to $L^{\infty}(G)$.

Proof. Let $\left(e_{\gamma}\right)$ be the bounded approximate identity of $L^{1}(G)$ in $P(G)$, and $m$ be a TIIM on $L U C(G)$. We choose an ultrafilter $\Gamma$ on the index set of $\left(e_{\gamma}\right)$ that dominates the order filter and define $M: L^{\infty}(G) \rightarrow \mathbb{C}$ by $M(f)=$ 
$\lim _{\Gamma} m\left(\frac{1}{\Delta} \tilde{e_{\gamma}} * f * \tilde{e_{\gamma}}\right) . M$ is a mean on $L^{\infty}(G)$. Now for fix $f \in L^{\infty}(G), \varphi \in P(G)$ and an arbitrary $\epsilon>0$ there exists $\gamma_{0}$ such that if $\gamma \geq \gamma_{0}$ then

$$
\left|M\left(\frac{1}{\Delta} \tilde{\varphi} * f\right)-m\left(\frac{1}{\Delta} \tilde{e_{\gamma}} * \frac{1}{\Delta} \tilde{\varphi} * f * \tilde{e_{\gamma}}\right)\right|<\epsilon
$$

and

$$
\left|M(f * \tilde{\varphi})-m\left(\frac{1}{\Delta} \tilde{e_{\gamma}} * f * \tilde{\varphi} * \tilde{e_{\gamma}}\right)\right|<\epsilon
$$

also we have

$$
\begin{gathered}
\lim m\left(\frac{1}{\Delta} \tilde{e_{\gamma}} * f * \tilde{\varphi}\right)=\lim m\left(\frac{1}{\Delta} \tilde{e_{\gamma}} * f * \tilde{e_{\gamma}} * \tilde{\varphi}\right)=\lim m\left(\frac{1}{\Delta} \tilde{\varphi} * \frac{1}{\Delta} \tilde{e_{\gamma}} * f * \tilde{e_{\gamma}}\right) \\
=\lim m\left(\frac{1}{\Delta} \tilde{\varphi} * f * \tilde{e_{\gamma}}\right)
\end{gathered}
$$

Now with (1), (2), (3) and some calculations we have

$$
\left|M\left(\frac{1}{\Delta} \tilde{\varphi} * f\right)-M(f * \tilde{\varphi})\right|<\epsilon
$$

Hence $M\left(\frac{1}{\Delta} \tilde{\varphi} * f\right)=M(f * \tilde{\varphi})$ and so $M$ is a TIIM. Also for $f \in L U C(G)$ we have $\frac{1}{\Delta} \tilde{e_{\gamma}} * f * \tilde{e_{\gamma}} \rightarrow f\left(\right.$ in $\left.\|\cdot\|_{u}\right)$, Therefore $M$ is an extention of $m$.

Now we give a simpler proof based on the technic used in [7,Th.3.2] for the following well known theorem, see [9].

Theorem 3. Let $G$ be a locally compact group. If $G$ is amenable, then $G$ is topological amenable.

Proof. By amenability of $G$ there exists a net $\left\{\psi_{\alpha}\right\} \subset P(G)$ such that $\lim _{\alpha}\left\|_{y}\left(\psi_{\alpha}\right)-\psi_{\alpha}\right\|_{1}=0$ uniformly on compacta; see [9]. Let $\varphi \in P(G)$ with $K=\operatorname{Supp}(\varphi)$ compact. For every $\epsilon>0$ we can find $\alpha_{0}$ such that if $\alpha \geq \alpha_{0}$, then $\left\|y\left(\psi_{\alpha}\right)-\psi_{\alpha}\right\|_{1}<\epsilon$ for all $y \in K^{-1}$. Hence we have

$$
\begin{gathered}
\left\|\varphi * \psi_{\alpha}-\psi_{\alpha}\right\|_{1}=\int_{G}\left|\int_{G} \Delta\left(y^{-1}\right) \varphi\left(y^{-1}\right) \psi_{\alpha}(y x) d y-\int_{G} \Delta\left(y^{-1}\right) \varphi\left(y^{-1}\right) \psi_{\alpha}(x) d y\right| d x \\
\quad \leq \int_{G}\|\|_{y}\left(\psi_{\alpha}\right)-\psi_{\alpha} \|_{1} \Delta\left(y^{-1}\right) \varphi\left(y^{-1}\right) d y \\
=\int_{K^{-1}}\|\|_{y}\left(\psi_{\alpha}\right)-\psi_{\alpha} \|_{1} \Delta\left(y^{-1}\right) \varphi\left(y^{-1}\right) d y<\epsilon
\end{gathered}
$$

Therefore $\lim _{\alpha}\left\|\varphi * \psi_{\alpha}-\psi_{\alpha}\right\|_{1}=0$ and hence we have $\lim _{\alpha}\left\|\varphi * \psi_{\alpha}-\psi_{\alpha}\right\|_{1}=0$ for all $\varphi \in P(G)$, that is $G$ is topological amenable. 


\section{References}

[1] C. A. Akemann, Operator Algebras Associated with fuchian Groups, Houston J. Math. 7(1981), 295-301.

[2] E. Bedos et P. Dela Harpe, Moyennabilitie Interieure Des Groups: Definitions et Examples, L Enseig Math. 32(1986), 139-157.

[3] E. Effros, Property $\Gamma$ And Inner Amenability, Proc. Amer. Math. Soc, Vol 47, No 2, 483-486 (1975).

[4] E. Hewitt and K.A. Ross. Abstract Harmonic Analysis (I), Springer-Verlag, New York, (1979).

[5] J.M. Ling, Inner Amenable Semigroups I, J. Math. Soc. Japan, Vol 49, No . 3, 603-616 (1997).

[6] V. Losert and H. Rindler, Asymptotically Central Functions and Invariant Extensions of Dirac Measures, Probability Measures on Groups, Proc. Conf. Oberwolfach (1983).

[7] R. Memarbashi and A. Riazi, Topological Inner Invariant Means, Studia. Sci. Math. Hungarica, 40 (2003), 293-299.

[8] R. Nasr-Isfahani, Inner Amenabilty of Lau Algebras, Arch. Math. (Brno) 37 (2001), 45-55.

[9] A. L. T. Paterson, Amenability, Mathematical Surveys and Monographs, Amer. Math. Soc. Providence 29 (1998).

Received: July 12, 2006 